\title{
Effect of muscle relaxation on the
} using an isolated forearm technique

\author{
Ka Young Rhee ${ }^{1}$, Tae-Yop Kim ${ }^{1}$, In Su $\mathrm{Oh}^{1}$, Seoung Joon $\mathrm{Lee}^{2}$, and \\ Thomas Ledowski ${ }^{3}$ \\ ${ }^{1}$ Department of Anesthesiology and Pain Medicine, Konkuk University Medical Center, ${ }^{2}$ Department of \\ Orthopedics, Konkuk University Medical Center, Seoul, Korea, ${ }^{3}$ School of Medicine and Pharmacology, University \\ of Western Australia, Perth, Australia
}

\begin{abstract}
Background: Total oxygen consumption has been found to be reduced under deep neuromuscular blockade due to a lower rate of metabolism of skeletal muscles. However, the magnitude of this effect in individual muscles has not been investigated. Thus the aim of this study was to compare the oxygenation of paralyzed versus non-paralyzed forearm muscle under tourniquet-provoked ischemia.

Methods: After ethics approval and written informed consent, 30 patients scheduled for elective hand and wrist surgery were included. Ischemia was provoked by inflation of bilateral upper arm tourniquets and muscle relaxation was achieved via intravenous administration of rocuronium $0.9 \mathrm{mg} / \mathrm{kg}$. Bilateral tourniquets were applied to both upper arms before induction of anesthesia and near infrared spectrometry (NIRS) electrodes applied on both forearms. Muscular ischemia in an isolated (= non-paralyzed, NP) as well as a paralyzed forearm (P) was created by sequential inflation of both tourniquets before and after intravenous administration of rocuronium. Muscle oxygen saturations $\left(\mathrm{S}_{\mathrm{m}} \mathrm{O}_{2}\right)$ of NIRS in both forearms and their changes were determined and compared.

Results: Data of 30 patients ( 15 male, 15 female; $41.8 \pm 14.7$ years) were analyzed. The speed of $\mathrm{S}_{\mathrm{m}} \mathrm{O}_{2}$ decrease $(50 \%$ decrease of $\mathrm{S}_{\mathrm{m}} \mathrm{O}_{2}$ from baseline (median [percentiles]: NP $210 \mathrm{~s}$ [180/480s] vs. P 180 [180/300]) as well as the maximum decrease in $\mathrm{S}_{\mathrm{m}} \mathrm{O}_{2}$ (minimum $\mathrm{S}_{\mathrm{m}} \mathrm{O}_{2}$ in \% (median [percentiles]: NP 20 [19/24] vs. P 21 [19/28]) were not significantly affected by neuromuscular paralysis.

Conclusions: No significant effect of muscle relaxation on NIRS-assessed muscle oxygenation under tourniquet-induced ischemia was found in human forearm muscles. (Korean J Anesthesiol 2015; 68: 13-16)
\end{abstract}

Key Words: Neuromuscular blockade, Near-infrared spectrometry, Skeletal muscles, Tourniquets.

Corresponding author: Thomas Ledowski, M.D., Ph.D.

School of Medicine and Pharmacology, University of Western Australia, Level 2 Royal Perth Hospital MRF Building, Rear 50 Murray Street, Perth WA 6000, Australia

Tel: 61-8-9224-0201, Fax: 61-8-9224020, E-mail: Thomas.ledowski@health.wa.gov.au

The content of this manuscript has been presented as a poster at the German Meeting of Anaesthesiologists (DAK) in Leipzig, Germany in May 2014. The work described in this manuscript was carried out at the Konkuk University Medical Centre in Seoul, Republic of Korea.

Received: July 16,2014. Revised: August 28, 2014. Accepted: October 16, 2014.

Korean J Anesthesiol 2015 February 68(1): 13-16

http://dx.doi.org/10.4097/kjae.2015.68.1.13

(c) This is an open-access article distributed under the terms of the Creative Commons Attribution Non-Commercial License (http://creativecommons.org/ licenses/by-nc/3.0/), which permits unrestricted non-commercial use, distribution, and reproduction in any medium, provided the original work is properly cited. 


\section{Introduction}

Neuromuscular blocking agents (NMBA) may reduce total oxygen consumption in intensive care patients via a reduction in oxidative muscle metabolism [1]. This effect could potentially serve as a protective mechanism during iatrogenic ischemia, such as surgery requiring a tourniquet for a prolonged period of time or in the case of free muscle flap transplantation.

However, no data exists regarding the dimension of this effect in individual muscle groups. Hence it was aim of this study to investigate the speed as well as the depth of muscle deoxygenation in paralyzed versus non-paralyzed muscle during tourniquet-induced ischemia.

\section{Meterials and Methods}

After approval by the Konkuk University Hospital Institutional Review Board (KUH1160059) on August 2013, registration with the Korean Trials Registry (KCT0001083) and written informed consent, 30 patients scheduled for elective hand and wrist surgery under general anesthesia were included in the study. Exclusion criteria included minors, patients' incapacity to consent, increased risk for thrombo-embolic events, co-morbidities or medication known or suspected to interact with the trial monitoring and allergic reactions to rocuronium. Prior to anaesthesia induction, 2 tourniquets were applied to both upper arms but not inflated. After induction of anesthesia with propofol 2-3

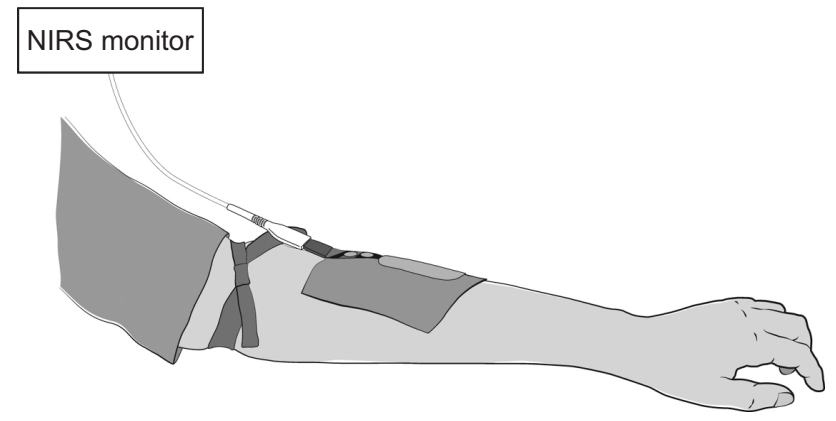

Fig. 1. The Near infrared spectrometry (NIRS) electrode was applied to the skin above the extensor digitorum muscle. $\mathrm{mg} / \mathrm{kg}$ and remifentanil $0.25-0.5 \mu \mathrm{g} / \mathrm{kg} / \mathrm{min}$ via an intravenous cannula inserted into the foot, patients were tracheally intubated and anesthesia was maintained with remifentanil 0.1-0.2 $\mu \mathrm{g} /$ $\mathrm{kg} / \mathrm{min}$ and sevoflurane $0.8-1.3 \mathrm{MAC}$, as appropriate. On both forearms, a near infrared spectrometry (NIRS) electrode was bilaterally applied to the skin above the extensor digitorum muscle and connected via the two entry connections to the NIRS monitor (Invos; Covidien, Dublin, Ireland) to assess the oxygen saturation of the muscle $\left(\mathrm{S}_{\mathrm{m}} \mathrm{O}_{2}\right)$ and surrounding tissues every 30 seconds. Thereafter, the first tourniquet was inflated to $300 \mathrm{mmHg}$ and a stopwatch started to record the duration of ischemia. Then rocuronium $0.9 \mathrm{mg} / \mathrm{kg}$ was administered. After confirmation of full neuromuscular paralysis via quantitative relaxometry, but within 5 minutes from first tourniquet inflation, the second tourniquet was inflated and the duration of ischemia monitored. By this means an isolated (non-paralyzed; NP) forearm was created while the other forearm was deeply paralyzed (P). Via the timing of ischemia duration and NIRS monitoring we assessed the time to a $50 \%$ decrease of $\mathrm{S}_{\mathrm{m}} \mathrm{O}_{2}$ (from baseline) and to the lowest $\mathrm{S}_{\mathrm{m}} \mathrm{O}_{2}$, as well as the lowest values for $\mathrm{S}_{\mathrm{m}} \mathrm{O}_{2}(\%$ from baseline and absolute) on both forearms (setup, see Fig. 1). The "lowest $\mathrm{S}_{\mathrm{m}} \mathrm{O}_{2}$ " was defined by the minimum value for $\mathrm{S}_{\mathrm{m}} \mathrm{O}_{2}$ that remained unchanged for at least 10 minutes.

\section{Statistics}

As no related data exists, the study was planned as a pilot project without a formal sample size calculation. At completion of data collection, all data were tested for normal distribution (Kolmogorov-Smirnov test) and are displayed as either mean \pm standard variation (SD) or median (25\%/75\% percentile), as appropriate. For comparison of the primary outcome parameters between both arms the Wilcoxon rank sum test was used.

\section{Results}

Data of 30 patients (15 male, 15 female; $41.8 \pm 14.7$ years; BMI $24.4 \pm 2.9$ ) were analyzed. However, neither the speed nor the depth of $\mathrm{S}_{\mathrm{m}} \mathrm{O}_{2}$ decrease was significantly different between the paralyzed and the non-paralyzed arm (Table 1).

Table 1. Comparison of the Muscular Oxygenation $\left(\mathrm{S}_{\mathrm{m}} \mathrm{O}_{2}\right)$ between the Paralyzed and the Non-paralyzed Forearm under Tourniquet-induced Ischemia

\begin{tabular}{lccc}
\hline \multicolumn{1}{c}{ Parameter } & Paralysed forearm & Non-paralysed forearm & P \\
\hline Time of $\mathrm{S}_{\mathrm{m}} \mathrm{O}_{2}$ decrease to 50\% from baseline (s) & $180(180 / 300)$ & $210(180 / 480)$ & 0.454 \\
Time of $\mathrm{S}_{\mathrm{m}} \mathrm{O}_{2}$ to minimum absolute value (s) & $480(270 / 1575)$ & $630(270 / 2497)$ & 0.449 \\
Minimum $\mathrm{S}_{\mathrm{m}} \mathrm{O}_{2}$ value (\% from baseline) (\%) & $21(19 / 28)$ & $20(19 / 24)$ & 0.160 \\
Minimum $\mathrm{S}_{\mathrm{m}} \mathrm{O}_{2}$ value (absolute value) (\%) & $15(15 / 15)$ & $15(15 / 15)$ & 0.953 \\
\hline
\end{tabular}

Data are displayed as median (25\%/75\% percentiles). 


\section{Discussion}

We did not find any significant difference in muscle oxygenation under tourniquet- induced ischemia when comparing paralyzed and non-paralyzed forearms of patients. Thus our results on first sight contradict earlier findings of a significant reduction in oxygen consumption after neuromuscular paralysis in intensive care patients [1]. However, even in their study, the observed difference in oxygen consumption before and after muscle relaxation was relatively small: $8.7 \pm 1.7 \%$. As in this previous study all skeletal muscles of one patient were either paralyzed or non-paralyzed, the contribution of a single muscle group (i.e. forearm muscles as in our study) has likely been much smaller and probably below the threshold of detection. Though the oxygen-sparing effect of neuromuscular paralysis may be low, the effect has consistently been described by several authors [1-3]. In contrast, Freebaim et al. [4] did not find any change of total oxygen consumption or gastric intra-mucosal $\mathrm{pH}$ after muscle relaxation when investigating 18 patients with severe septic shock. Whether the pathophysiology of sepsis contributed to these contradictory findings remains speculative but may have played a role.

However, even small effects of muscle relaxation on oxygen consumption could become important in the context of temporary ischemia, such as during surgery with a tourniquet or in free muscle flap transplants. In the latter, a clear effect of ischemic time on flap survival has been demonstrated [5]. Small decreases in muscular oxygen consumption and therefore the preservation of viable oxygen saturation levels for a prolonged period of time could thus at least hypothetically increase the odds for muscle flap survival. However, with the method used by us we failed to demonstrate a marked effect of muscle relaxation on muscle oxygenation under ischemia. It is beyond the scope of our limited experiment to conclude about the reasons for the contradictory results. However, some may be found in the limitations of our study, with the most obvious limitation being the relatively small sample size. Especially in the light of the expected differences being small, 30 patients may have been an insufficient sample to demonstrate the anticipated effect. In addition were tourniquets not inflated fully simultaneously but within approx. 5 minutes from each other due to the need to administer rocuronium after inflation of the first tourniquet. However, with anesthesia at steady state (i.e. hemoglobin, inspired $\mathrm{O}_{2}$ fraction, ventilator settings, sevoflurane and remifentanil concentrations) an effect on the reported results appears unlikely. Furthermore is the method of NIRS monitoring controversially discussed. Crenshaw et al. [6] reported the reliability of NIRS measured muscle oxygenation to not only vary greatly between investigated upper extremity muscles but to be also significantly influenced by factors such as ischemia or hyperemia. Furthermore were baseline values of $\mathrm{S}_{\mathrm{m}} \mathrm{O}_{2}$ frequently different between the two arms of individual patients. Though it is unclear whether this could have affected the percentage change of NIRS values in the time course of ischemia, this cannot be fully excluded. The latter certainly questions NIRS monitoring as the method of choice in our study. However, we were unable to identify a suitable alternative with similar benefits to NIRS (i.e. non-invasiveness; simultaneous assessment of oxygenation of two muscles [both forearms] via two channels of one monitor). In addition did other authors find NIRS to be both sensitive and specific to measure oxygenation levels in single compartments of the forearm [7].

We conclude that, though an oxygen sparing effect of muscle relaxation has previously been demonstrated, we failed to find differences in NIRS measured oxygenation comparing paralyzed vs. non-paralyzed muscles under tourniquet-induced ischemia of the forearm. As a protective effect of muscle relaxation under such conditions is at least plausible and may be clinically relevant, further research of the topic is warranted.

\section{Acknowledgments}

Thomas Ledowski has consulted for and accepted a research grant from MSD, the manufacturer of rocuronium, the muscle relaxant used in the study. However, none of the aforementioned has been related to this publication, and MSD has not in any form been involved in planning, analysis or manuscript preparation. The current study has been entirely funded by the University of Western Australia.

\section{References}

1. Vernon DD, Witte MK. Effect of neuromuscular blockade on oxygen consumption and energy expenditure in sedated, mechanically ventilated children. Crit Care Med 2000; 28: 1569-71.

2. Steinhorn DM. Neuromuscular blockade provides no benefit over adequate sedation in ventilated dogs. J Crit Care 1995; 10: 45-50.

3. Marik PE, Kaufman D. The effects of neuromuscular paralysis on systemic and splanchnic oxygen utilization in mechanically ventilated patients. Chest 1996; 109: 1038-42.

4. Freebairn RC, Derrick J, Gomersall CD, Young RJ, Joynt GM. Oxygen delivery, oxygen consumption, and gastric intramucosal pH are not 
improved by a computer-controlled, closed-loop, vecuronium infusion in severe sepsis and septic shock. Crit Care Med 1997; 25: 72-7.

5. Nishikawa H, Manek S, Barnett SS, Charlett A, Green CJ. Pathology of warm ischaemia and reperfusion injury in adipomusculocutaneous flaps. Int J Exp Pathol 1993; 74: 35-44.

6. Crenshaw AG, Elcadi GH, Hellstrom F, Mathiassen SE. Reliability of near infrared spectroscopy for measuring forearm and shoulder oxygenation in healthy males and females. Eur J Appl Physiol 2012; 112: 2703-15.

7. Cola AL, Herman RA Jr, Heimlich JB, Ahsan S, Freedman BA, Shuler MS. Ability of near infrared spectroscopy to measure oxygenation in isolated upper extremity muscle compartments. J Hand Surg Am 2012; 37: 297-302. 